\title{
Development of a Universal Anti-Polyethylene Glycol Reporter Gene for Noninvasive Imaging of PEGylated Probes
}

\author{
Kuo-Hsiang Chuang*1, Hsin-Ell Wang*2, Ta-Chun Cheng ${ }^{1}$, Shey-Cherng Tzou ${ }^{3}$, Wei-Lung Tseng ${ }^{4,5}$, \\ Wen-Chun Hung ${ }^{5,6}$, Ming-Hong Tai ${ }^{5,6}$, Tien-Kuei Chang ${ }^{2}$, Steve R. Roffler ${ }^{7}$, and Tian-Lu Cheng ${ }^{3,5}$ \\ ${ }^{1}$ Graduate Institute of Medicine, Kaohsiung Medical University, Kaohsiung, Taiwan; ${ }^{2}$ Institute of Radiological Science, National \\ Yang-Ming University, Taipei, Taiwan; ${ }^{3}$ Department of Biomedical Science and Environmental Biology, Kaohsiung Medical \\ University, Kaohsiung, Taiwan; ${ }^{4}$ Department of Chemistry, National Sun Yat-sen University, Kaohsiung, Taiwan; ${ }^{5}$ National Sun \\ Yat-Sen University-Kaohsiung Medical University Joint Research Center, Kaohsiung, Taiwan; 'Institute of Biomedical Sciences, \\ National Sun Yat-Sen University, Kaohsiung, Taiwan; and ${ }^{7}$ Institute of Biomedical Sciences, Academia Sinica, Taipei, Taiwan
}

A reporter gene can provide important information regarding the specificity and efficacy of gene or cell therapies. Although reporter genes are increasingly used in experimental and clinical studies, a highly specific yet nonimmunogenic reporter that can track genes and cells in vivo by multiple imaging technologies still awaits development. In this study, we constructed a versatile and nonimmunogenic reporter gene to noninvasively image gene expression or cell delivery by optical imaging, MRI, and small-animal PET. Methods: We cloned and expressed a membrane-anchored anti-polyethylene glycol (PEG) reporter that consists of the Fab fragment of a mouse anti-PEG monoclonal antibody, AGP3, fused to the C-like extracellular-transmembrane-cytosolic domains of the mouse B7-1 receptor. Binding of PEGylated probes (PEG-NIR797 for optical imaging, PEGsuperparamagnetic iron oxide for MRI, and ${ }^{124} \mathrm{I}-\mathrm{PEG}$ for smallanimal PET) were examined in vitro and in vivo. In addition, we compared the specificity, immunogenicity, and probe toxicity of the anti-PEG reporter with the gold standard reporter gene, type 1 herpes simplex virus thymidine kinase (HSV-tk). Finally, we derived a humanized anti-PEG reporter and evaluated its imaging function in vivo with subcutaneous and metastatic tumor models in mice. Results: The cells or tumors that stably expressed anti-PEG reporters selectively accumulated various PEGylated imaging probes and could be detected by optical imaging, MRI, and small-animal PET. Importantly, the anti-PEG reporter displayed an imaging specificity comparable to the HSV-tk reporter but did not provoke immune responses or cause toxicity to the host. Furthermore, the humanized anti-PEG reporter retained high imaging specificity in vivo. Conclusion: The highly specific and nonimmunogenic anti-PEG reporter may be paired with PEGylated probes to provide a valuable system to image

Received Oct. 26, 2009; revision accepted Feb. 17, 2010.

For correspondence or reprints contact either of the following:

Tian-Lu Cheng, Faculty of Biomedical and Environmental Biology,

Kaohsiung Medical University, 100 Shih-Chuan 1st Rd., Kaohsiung,

Taiwan.

E-mail: tlcheng@kmu.edu.tw

Steve Roffler, Institute of Biomedical Sciences, Academia Sinica,

Academia Rd., Section 2, No. 128, Taipei 11529, Taiwan.

E-mail: sroff@ibms.sinica.edu.tw

${ }^{*}$ Contributed equally to this work.

COPYRIGHT ๑ 2010 by the Society of Nuclear Medicine, Inc. gene expression or cell delivery in experimental and clinical studies.

Key Words: polyethylene glycol; anti-PEG reporter; humanized anti-PEG reporter; PEGylated imaging probes; noninvasive imaging

J Nucl Med 2010; 51:933-941

DOI: 10.2967/jnumed.109.071977

$\mathbf{N}$ oninvasive imaging of reporter genes can provide information regarding the expression, location, and persistence of genes and cells in vivo. Ideally, the reporter gene should allow specific imaging but display minimal immunogenicity. Type 1 herpes simplex virus thymidine kinase (HSV-tk), regarded as a gold standard reporter gene, allows specific imaging of gene expression or transplanted cells by SPECT (1) and PET (2). Unfortunately, the immunogenicity of HSV-tk (3) and the mutagenic potential of its probes (4) may limit its experimental and clinical use. Endogenous reporter genes such as the dopamine $\mathrm{D}_{2}$ receptor (5) and the transferrin receptor (6) display low immunogenicity, but the fact that the $\mathrm{D}_{2}$ receptor is also expressed in the brain stratum and in the pituitary glands (7) and the transferrin receptor is widely expressed in vivo (8) may limit their specificity. An optimal reporter gene that possesses high imaging specificity and low immunogenicity remains to be developed for human use.

Membrane-anchored antibodies are attractive for the development of highly specific and nonimmunogenic reporter genes (9-12). Antibody reporters can be designed to exhibit low immunogenicity by using antibodies derived from the species of interest. Humanized antibodies can minimize both humoral and cellular immune responses to allow repeated and persistent imaging of gene expression in humans. Furthermore, antibody-antigen pairs possess high specificity and affinity without interference from cellular factors. 
We have previously generated an anti-polyethylene glycol (PEG) antibody that binds the repeating backbone $\left(\mathrm{O}-\mathrm{CH}_{2}-\mathrm{CH}_{2}\right)_{34}$ of PEG and can detect or clear PEGylated proteins, liposomes, and nanoparticles in vitro and in vivo (13). PEG is a water-soluble, nontoxic, nonantigenic, and biocompatible polymer that has been approved by the Food and Drug Administration for human use (14). PEGylation is a proven technology; many PEGylated probes, such as PEGylated superparamagnetic iron oxide (15), PEGylated fluorescent probes (16), and PEGylated chelating agents (17), are in commercial or late-stage clinical trials. As the list of PEGylated probes continuously expands, an imaging strategy that uses PEGylated probes to image human or humanized anti-PEG reporters should possess great potential for clinical applications.

In this study, we developed a versatile and universal reporter based on the surface expression of an anti-PEG Fab fragment on cells (Fig. 1A). We examined the surface expression and the function of the anti-PEG reporter and tested whether the anti-PEG reporter could be detected by optical imaging, MRI, or small-animal PET. We compared the imaging specificity, immunogenicity, and probe toxicity of the anti-PEG reporter with those of the HSV-tk reporter in mice. Finally, we tested if the humanized anti-PEG reporter could be used for in vivo imaging of subcutaneous and metastatic tumor models by small-animal PET and optical imaging, respectively. The humanized anti-PEG reporter imaging system is expected to provide a valuable tool for monitoring gene and cell delivery in humans.

\section{MATERIALS AND METHODS}

\section{Cells and Animals}

NIH 3T3 mouse fibroblasts and human HeLa cervical cancer cells were purchased from American Type Culture Collection. EJ human bladder carcinoma cells and CNL Chang liver cells were obtained from Academia Sinica. The cells were cultured in Dulbecco minimal essential medium (Sigma-Aldrich) supplemented with $10 \%$ heat-inactivated bovine calf serum, penicillin (100 units $/ \mathrm{mL})$, and streptomycin $(100 \mu \mathrm{g} / \mathrm{mL})$ at $37^{\circ} \mathrm{C}$ in a humidified atmosphere of $5 \% \mathrm{CO}_{2}$. Female BALB/c nude mice (6-8 wk old) and female $\mathrm{BALB} / \mathrm{c}$ mice (6-8 wk old) were obtained from the National Laboratory Animal Center, Taipei, Taiwan. All mice receiving radioiodinated probes were pretreated with Lugol solution (Sigma) in their drinking water for $2 \mathrm{~d}$ to block thyroidal uptake of free radioiodine (18). All animal experiments were performed in accordance with institutional guidelines and approved by the Animal Care and Use Committee of the Kaohsiung Medical University.

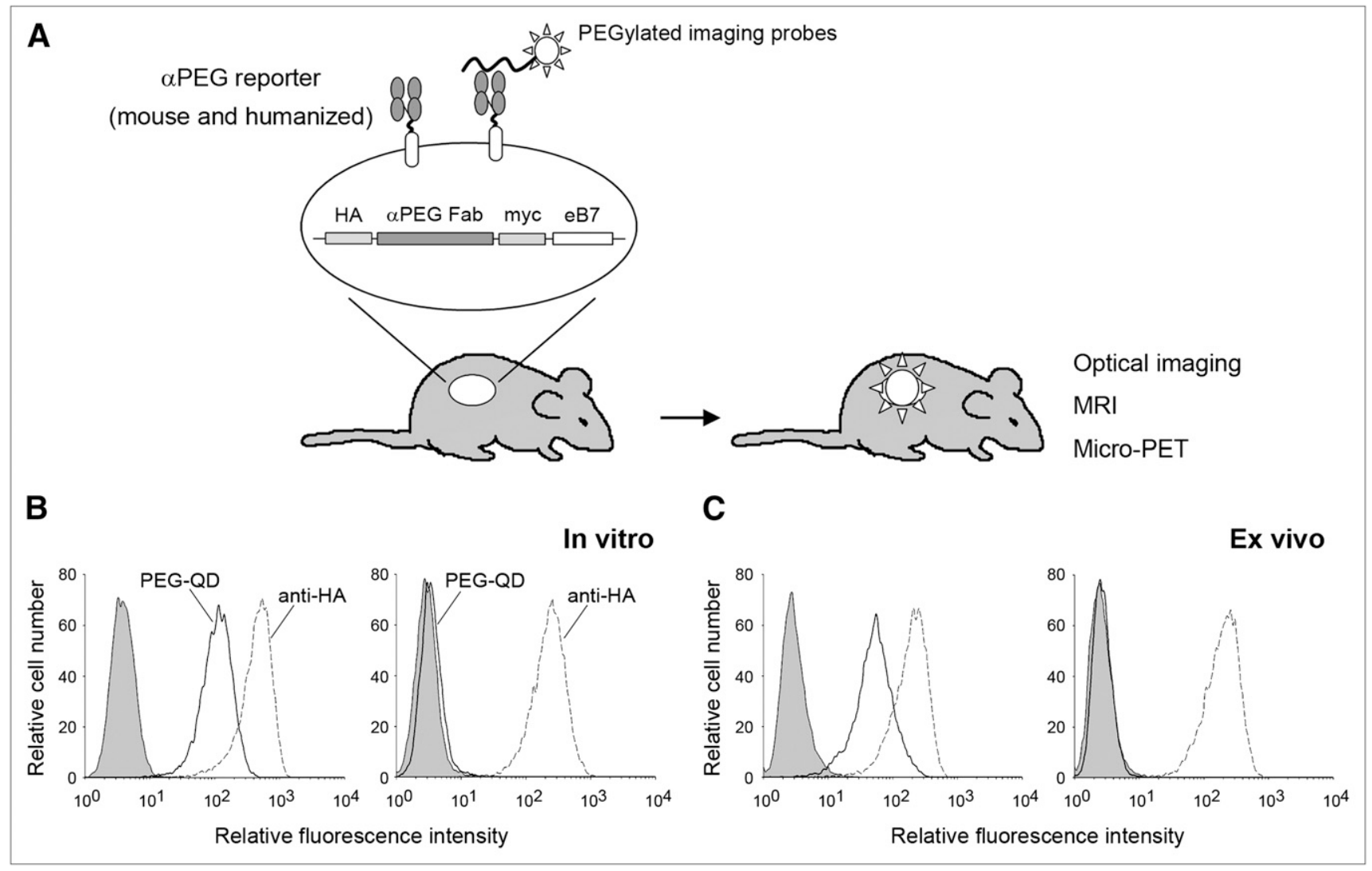

FIGURE 1. Anti-PEG reporter gene system. (A) Strategy and organization of anti-PEG reporter gene. (B and C) Flow cytometric analysis of surface expression (dashed lines, stained by hemagglutinin antibody) and function (solid lines, stained by PEGquantum dot) of anti-PEG (left graph) or anti-DNS (right graph) reporters in vitro (B) and ex vivo (C). Shaded areas of graphs show mock staining with PBS. $\alpha \mathrm{PEG}=$ anti-PEG; $\mathrm{HA}=$ hemagglutinin; myc = c-myc epitope; $\mathrm{QD}=$ quantum dot. 
Generation of Anti-PEG Reporter-Expressing Cells

The $\mathrm{V}_{\mathrm{L}}-\mathrm{C}_{\kappa}$ and $\mathrm{V}_{\mathrm{H}^{-}}-\mathrm{C}_{\mathrm{H}} 1$ domains of the anti-PEG reporter were cloned from complementary DNA prepared from the AGP3 hybridoma (19) following a previously described method (10). Primers used in the cloning of $\mathrm{V}_{\mathrm{L}}-\mathrm{C \kappa}$ and $\mathrm{V}_{\mathrm{H}^{-}} \mathrm{C}_{\mathrm{H}} 1$ are as follows: $\mathrm{V}_{\mathrm{L}}-\mathrm{CK}$ sense: $5^{\prime}$-tgctggggeccagccggccgatattgtgttgacgcaggct-3';

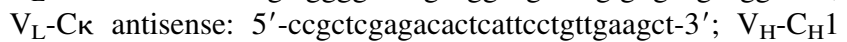
sense: $5^{\prime}$-gaagatctgaagtgcagctggtggagtct- $3^{\prime}$; and $\mathrm{V}_{\mathrm{H}^{-}} \mathrm{C}_{\mathrm{H}} 1$ antisense: 5'-caggtcgacagctggaatgggcacatgcag-3'. The $\mathrm{V}_{\mathrm{L}}-\mathrm{C} \kappa$ and $\mathrm{V}_{\mathrm{H}^{-}} \mathrm{C}_{\mathrm{H}} 1$ genes were joined by a composite furin-2A protease cleavage site (20) and fused to the complementary DNA sequence encoding the C-like extracellular-transmembrane-cytosolic domains of the mouse B7-1 antigen (eB7) in the pLNCX-eB7 retroviral vector (10) to generate pLNCX-anti-PEG-eB7. A plasmid (pLNCX-anti-DNS-eB7) that encoded a membrane Fab with specificity for 5-(dimethylamino)naphthalene-1-sulfonyl chloride (DNS) (21) was constructed in an analogous fashion. EJ/anti-PEG or EJ/anti-DNS cells that stably expressed approximately equivalent levels of anti-PEG or anti-DNS reporters were generated by retroviral transduction as previously described (22). Characterization of the EJ/anti-PEG or EJ/anti-DNS cells by Western blotting and by flow cytometry is described in the Supplemental Methods (supplemental materials are available online only at http://jnm.snmjournals.org).

\section{Synthesis of PEG-NIR797 and 4-Arm ${ }^{124}$ |-PEG-SHPP, ${ }^{124}$ I-FIAU Imaging Probes}

The protocols of probe syntheses are provided in the Supplemental Methods. The 4-arm ${ }^{124} \mathrm{I}-\mathrm{PEG}-\mathrm{N}$-succinimidyl-3-(4hydroxyphenyl)propionate (SHPP) and ${ }^{124} \mathrm{I}-2^{\prime}$-fluoro- 2 '-deoxybeta-D-arabinofuranosyl-5-iodouracil (FIAU) were more than 95\% pure, with specific activities calculated to be 27.8 and 1.35 $\mathrm{GBq} / \mu \mathrm{mol}$, respectively. The half-life of 4 -arm ${ }^{124} \mathrm{I}$-PEG-SHPP was about $8.2 \mathrm{~min}$.

\section{Optical Imaging of Anti-PEG Reporter In Vitro and In Vivo}

EJ/anti-PEG and EJ/anti-DNS cells $\left(5 \times 10^{5}\right)$ were stained with $0.02,0.1$, and $0.5 \mu \mathrm{M}$ PEG-NIR797 in phosphate-buffered saline (PBS) containing $0.05 \%$ bovine serum albumin on ice for $45 \mathrm{~min}$. The cells were washed with cold PBS, and the surface immunofluorescence was measured on an IVIS50 optical imaging system (excitation, $760 \mathrm{~nm}$; emission, $835 \mathrm{~nm}$ ) (Caliper Life Sciences). For in vivo imaging, BALB/c nude mice bearing established EJ/ anti-PEG and EJ/anti-DNS tumors $\left(200 \mathrm{~mm}^{3}\right)$ in their right and left hind leg regions, respectively, were intravenously injected with PEG-NIR797 fluorescent probes ( $2 \mathrm{mg} / \mathrm{kg}$ of body weight), and the optical images were acquired with an IVIS50 at 4, 24, and $48 \mathrm{~h}$ after injection. The regions of interest of tumor areas were drawn and analyzed with Living Image software, version 2.50 (Caliper Life Sciences).

\section{T2-Weighted MRI of Anti-PEG Reporter In Vitro and In Vivo}

EJ/anti-PEG and EJ/anti-DNS cells $\left(3 \times 10^{6}\right)$ were incubated with $48,24,12$, and $6 \mu \mathrm{M} \mathrm{PEG}_{2000}$ conjugated superparamagnetic iron oxide (PEG-SPIO, kindly provided by Yun-Ming Wang of the National Chiao Tung University, Taiwan) on ice for $30 \mathrm{~min}$. After being washed with cold PBS, the PEG-SPIO labeled cells were collected by centrifugation $(1,500 \mathrm{rpm})$ and imaged with a clinical 3.0-T MRI scanner (Sigma; GE Healthcare) equipped with a high- resolution head coil. All samples were measured by a T2-weighted spin-echo sequence (repetition time, 2,500 ms; echo time, $90 \mathrm{~ms}$ ).

For in vivo imaging, BALB/c nude mice bearing established EJ/ anti-PEG and EJ/anti-DNS tumors $\left(300 \mathrm{~mm}^{3}\right)$ in their right and left hind leg regions, respectively, were intravenously injected with PEG-SPIO (10 mg of iron/kg of body weight). Pentobarbitalanesthetized mice were sequentially imaged at 1,2 , and $24 \mathrm{~h}$ after injection with a 7-T horizontal-bore magnet (Bruker Instruments). A T2-weighted spin-echo sequence (repetition time, 2,500 ms; echo time, $90 \mathrm{~ms}$ ) was used for MRI.

\section{In Vivo Imaging of Anti-PEG and HSV-tk Reporters by Small-Animal PET}

$\mathrm{BALB} / \mathrm{c}$ nude mice bearing established EJ/anti-PEG and EJ/antiDNS or EJ/TK (an EJ cell line expressing functional HSV-tk) tumors $\left(200 \mathrm{~mm}^{3}\right)$ in their right and left hind leg regions, respectively, were anesthetized with halothane vapor and then intravenously injected with $3,700 \mathrm{kBq}$ (in $100 \mu \mathrm{L}$ ) of 4-arm ${ }^{124}$ I-PEG-SHPP or ${ }^{124}$ I-FIAU. The mice were sequentially imaged at 4,24 , and $48 \mathrm{~h}$ after injection with a micro-PET R4 scanner (Concorde Microsystems), following an imaging protocol previously described (18).

\section{Immune Response to Anti-PEG and HSV-tk Reporter Proteins}

The immunogenicity of the anti-PEG and the HSV-tk reporters was compared using a previously described protocol (22). Briefly, $\mathrm{BALB} / \mathrm{c}$ mice were intravenously injected with $20 \mu \mathrm{g}$ of $\mathrm{pLNCX}$ (vector control, $n=6$ ), pLNCX-anti-PEG-eB7 $(n=8)$, or pLNCX-HSV-tk $(n=8)$. Preimmune and immune (day 10) sera (diluted 1:200 in PBS) were added to the wells of microtiter plates precoated with BALB 3T3 cells transfected with pLNCX, pLNCX-anti-PEG-eB7, or pLNCX-HSV-tk-eB7 (which codes for a membrane form of HSV-tk). Binding of antibodies on the cells was detected by enzyme-linked immunosorbent assay as previously described (22). All readings were background-adjusted by subtracting the absorbance of the wells incubated with preimmune sera.

\section{Construction of Humanized Anti-PEG Reporter}

The anti-PEG reporter was humanized using a previously reported protocol with minor modifications (23). Briefly, to select human framework sequences for complementarity-determiningregion grafting, we compared the $\mathrm{V}_{\mathrm{H}}$ and $\mathrm{V}_{\mathrm{L}}$ sequences of the anti-PEG Fab with the National Center for Biotechnology Information database of human immunoglobulin germline sequences in the variable and joining regions using the IgBLAST program (http://www.ncbi.nlm.nih.gov/igblast/). DP35-JH4 and A2c-JK2 were found to be the most homologous to the $V_{H}$ and $\mathrm{V}_{\mathrm{L}}$ of anti-PEG reporter, respectively. For construction of the humanized $\mathrm{V}_{\mathrm{L}}$, the complementarity-determining regions of antiPEG $\mathrm{V}_{\mathrm{L}}$, determined by the rule of Kabat et al. (24), was grafted onto human A2c-JK2. For construction of the humanized $\mathrm{V}_{\mathrm{H}}$, the complementarity-determining regions and 1 FR3 residue (Ser73) of anti-PEG $\mathrm{V}_{\mathrm{H}}$ were similarly grafted onto DP25-JH4. The humanized anti-PEG $\mathrm{V}_{\mathrm{L}}$ and $\mathrm{V}_{\mathrm{H}}$ segments were synthesized by assembly polymerase chain reaction (23). The pLNCX-human anti-PEG-eB7 plasmid and tumor cells expressing humanized anti-PEG reporters (EJ/human anti-PEG and Hela/human antiPEG) were constructed as described above. 


\section{In Vivo Imaging of Human Anti-PEG Reporter}

The human anti-PEG reporter was examined in both subcutaneous and metastatic tumor models in vivo. For imaging the subcutaneous tumor model, BALB/c nude mice $(n=3)$ bearing established EJ/human anti-PEG and EJ/anti-DNS tumors (200 $\mathrm{mm}^{3}$ ) in their right and left hind leg regions, respectively, were imaged by small-animal PET as described above.

For imaging the metastatic tumor model, BALB/c nude mice $(n=3)$ were intravenously injected with Hela or Hela/human antiPEG cells $\left(5 \times 10^{6}\right.$ cells $)$. After $3 \mathrm{wk}$, the mice were intravenously injected with PEG-NIR797 fluorescent probes $(2 \mathrm{mg} / \mathrm{kg}$ of body weight). Whole-body images and lung-tissue images of these mice were acquired by the IVIS50 optical imaging system (excitation, $760 \mathrm{~nm}$; emission, $835 \mathrm{~nm}$ ) at $48 \mathrm{~h}$ after injection.

\section{Statistical Analysis}

Differences in tumor uptake of PEG-NIR797 were compared by the signed rank test. Data were considered significant at a $P$ value of 0.05 or less. The cytotoxicity of iodinated 4-arm PEG-SHPP and FIAU and the immunogenicity of anti-PEG and HSV-tk reporters were compared by 1-way ANOVA using InStat software (version 3.0; GraphPad Software) followed by the Student $t$ test. Bonferroni adjustment was used to adjust the significance level for multiple (3) comparisons. A $P$ value of no more than the Bonferroni-corrected significance level $(0.05 / 3=0.0167)$ was considered significant.

\section{RESULTS}

\section{Characterization of Surface Anti-PEG Reporter}

We constructed a membrane-anchored Fab with specificity to PEG as a reporter gene, or anti-PEG reporter. The light chain $\left(\mathrm{V}_{\mathrm{L}}-\mathrm{C}_{\mathrm{K}}\right)$ is joined - through a furin cleavage site and $2 \mathrm{~A}$ peptide $(20)$ - to the heavy chain $\left(\mathrm{V}_{\mathrm{H}^{-}} \mathrm{C}_{\mathrm{H}} 1\right)$, which is itself fused to the $\mathrm{C}$-like extracellular-transmembranecytosolic domains of the mouse B7-1 antigen (Fig. 1A). A membrane-anchored Fab with specificity for DNS was constructed in an analogous fashion. The DNS Fab does not bind PEG and was used as a negative control reporter.

To analyze the anti-PEG and the control anti-DNS reporters, total cellular, membrane, and cytosolic proteins were separated by SDS-PAGE under reducing conditions and analyzed by Western blotting using antibodies to the hemagglutinin epitope present in the light chain (Supplemental Fig. 1A), or to the c-myc epitope present in the heavy chain (Supplemental Fig. 1B). Two protein bands with apparent molecular weights of approximately 26 and $50 \mathrm{kDa}$, corresponding to the light chain and the heavy chain of the antiPEG reporter, respectively, were detected. Most of the membrane-anchored anti-PEG reporters were present as fully cleaved Fab fragments. A minor band of approximately $84 \mathrm{kDa}$, representing uncleaved $\mathrm{V}_{\mathrm{L}}-\mathrm{C}_{\mathrm{K}}$-furin- $2 \mathrm{~A}-\mathrm{V}_{\mathrm{H}}-\mathrm{C}_{\mathrm{H}} 1-\mathrm{B} 7$, constituted only a small proportion of the fusion protein expressed on the cell membrane. These results indicate that correctly processed anti-PEG reporter was expressed on the plasma membrane. Similar expression of the antiDNS reporter on EJ cells was also noted.

EJ/anti-PEG and EJ/anti-DNS cells were stained with a commercial PEG-quantum dot to assess the PEG binding activity of the anti-PEG reporter. As shown in Figure 1B, EJ/anti-PEG cells but not EJ/anti-DNS cells selectively bound PEG-quantum dot. For testing of whether functional anti-PEG reporters can be expressed in vivo, cell suspensions prepared from freshly resected EJ/anti-PEG or EJ/ anti-DNS tumors were stained with a hemagglutinin antibody or PEG-quantum dot. Figure 1C shows that EJ/antiPEG and EJ/anti-DNS cells recovered from tumors retained expression of the respective reporters, but only EJ/anti-PEG cells displayed PEG-quantum dot binding activity. These results show that functional anti-PEG reporters were expressed on cell membranes in vitro and in vivo.

\section{In Vitro and In Vivo Imaging of Anti-PEG Reporter}

We tested 3 major mainstream imaging technologies for detecting the anti-PEG reporter: optical imaging, MRI, and PET. EJ/anti-PEG and EJ/anti-DNS cells were stained with PEG-NIR797, PEG-SPIO, or 4-arm ${ }^{124}$ I-PEG-SHPP. Probe binding on cells was quantified by optical imaging, T2weighted MRI, or $\gamma$-counting, respectively. Figure 2A shows quantitative binding of PEG-NIR797 to EJ/anti-PEG or EJ/ anti-DNS cells in vitro. On average, signal intensity (defined as photons $/ \mathrm{s} / \mathrm{cm}^{2}$ ) was 6.5-, 7.5-, and 9.8-fold stronger in the EJ/anti-PEG cells than in the EJ/anti-DNS cells after the addition of $0.5,0.1$, and $0.02 \mu \mathrm{M}$ PEG-NIR797, respectively. Similarly, PEG-SPIO was selectively trapped by the EJ/antiPEG cells at all concentrations examined (darker images in Fig. 2C). The T2 signal intensity was reduced by more than $23 \%$ (range, $\sim 23.1 \%-94.1 \%$ ) in EJ/anti-PEG cells, compared with EJ/anti-DNS cells. In line with the optical imaging and the MRI results, more than 55-fold (range, $\sim 55$ - to 159 -fold) of 4-arm ${ }^{124}$ I-PEG-SHPP was retained on EJ/anti-PEG cells than on EJ/anti-DNS cells (Fig. 2E). These data indicate that the anti-PEG reporter can trap various PEGylated probes for optical imaging, MRI, and PET.

To investigate whether the anti-PEG reporter could be noninvasively imaged in vivo, we intravenously injected PEG-NIR797, PEG-SPIO, or 4-arm ${ }^{124}$ I-PEG-SHPP into BALB/c nude mice bearing subcutaneous EJ/anti-PEG and EJ/anti-DNS tumors. Whole-body images of the tumorbearing mice were acquired by optical imaging, T2-weighted MRI, and small-animal PET, respectively. Figure 2B shows that PEG-NIR797 probe was selectively retained at EJ/antiPEG tumors but not at EJ/anti-DNS tumors. The nearinfrared signal lasted as long as $48 \mathrm{~h}$ after injection and clearly indicated the locations of the EJ/anti-PEG tumors. Fluorescent intensities in the region of interest were 9.0-, 10.9-, and 16.9-fold greater in the EJ/anti-PEG tumors than in the EJ/anti-DNS tumors at 4, 24, and $48 \mathrm{~h}$, respectively ( $P<0.028$ for all time points). Similarly, PEGSPIO was selectively retained in EJ/anti-PEG tumors, leading to markedly darker signals (a positive T2weighted signal) in the EJ/anti-PEG tumors but not in the control EJ/anti-DNS tumors (Fig. 2D). Finally, consistent with the in vitro tests, the 4-arm ${ }^{124}$ I-PEG-SHPP preferentially accumulated in EJ/anti-PEG tumors (Fig. 


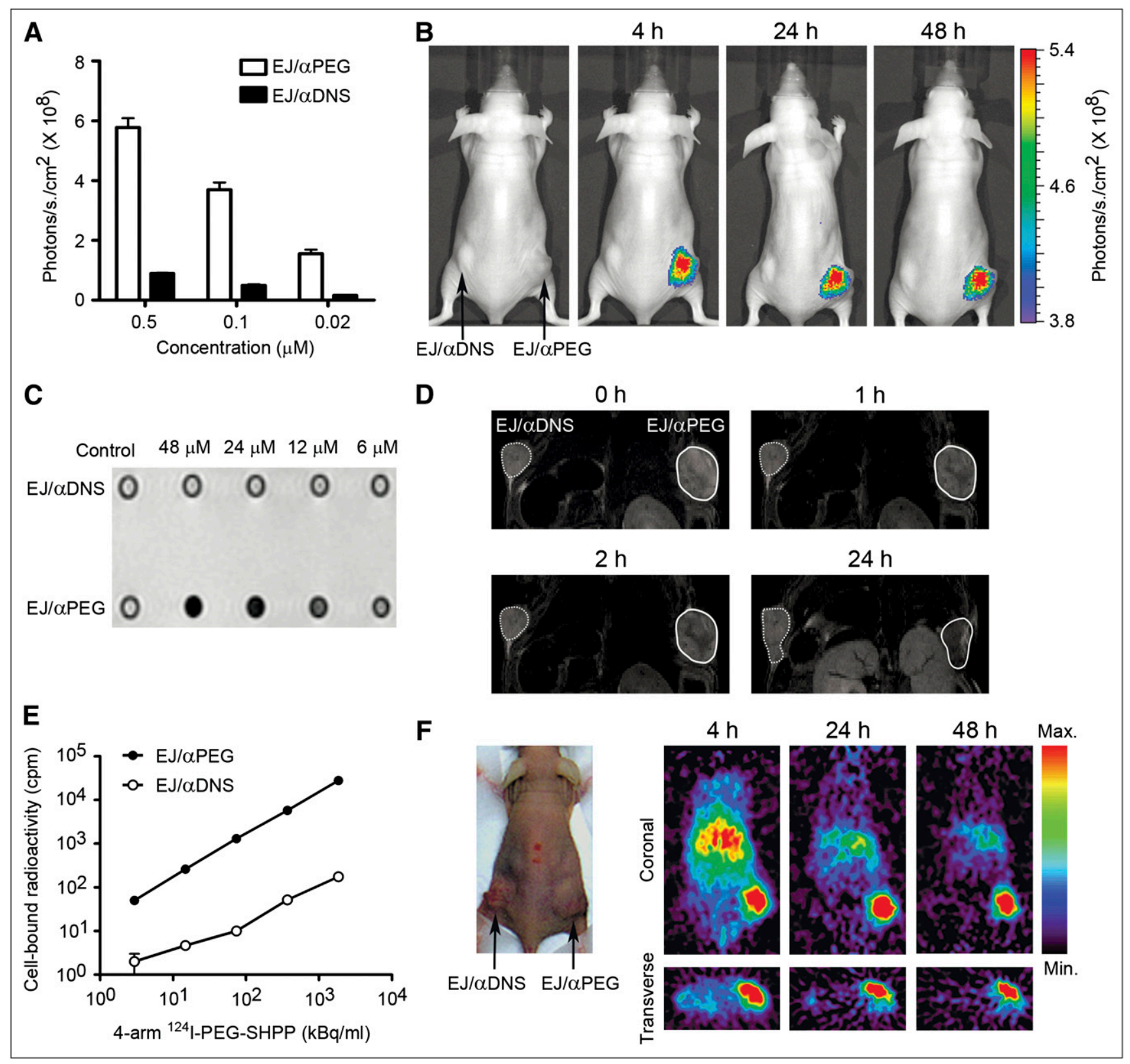

FIGURE 2. Imaging of anti-PEG reporter by multiple imaging systems. (A) In vitro optical imaging of EJ/anti-PEG cells or EJ/ anti-DNS cells stained with graded concentrations of PEG-NIR797, with SEM of triplicate determinations indicated. (B) In vivo optical imaging of mice bearing EJ/anti-PEG tumors (right hind legs) and EJ/anti-DNS tumors (left hind legs) at 4, 24, and 48 $\mathrm{h}$ after injection of PEG-NIR797. Representative images of 3 independent experiments $(n=6)$ are shown. (C) In vitro MRI of EJ/ anti-PEG cells (bottom) and EJ/anti-DNS cells (top) stained with graded concentrations of PEG-SPIO. (D) In vivo MRI of mice bearing EJ/anti-PEG tumors (solid line) and EJ/anti-DNS tumors (dashed line) at 1, 2, and $24 \mathrm{~h}$ after injection of PEG-SPIO (10 $\mathrm{mg}$ of iron/ $\mathrm{kg}$ of body weight). Note that images of EJ/anti-PEG tumors are darker. Representative images of 3 independent experiments $(n=3)$ are shown. (E) In vitro binding of 4 -arm ${ }^{124}$ I-PEG-SHPP to EJ/anti-PEG cells or EJ/anti-DNS cells after staining with graded concentrations of 4 -arm ${ }^{124}$ I-PEG-SHPP. (F) In vivo small-animal PET of mice bearing EJ/anti-PEG tumors (right hind legs) and EJ/anti-DNS tumors (left hind legs) at 4, 24, and $48 \mathrm{~h}$ after injection of 4-arm ${ }^{124}$ I-PEG-SHPP. Small-animal PET images were acquired in coronal and transverse planes. Representative images of 3 independent experiments $(n=3)$ are shown. $\alpha$ PEG $=$ anti-PEG; $\alpha$ DNS $=$ anti-DNS.

2F). On average, EJ/anti-PEG tumors accumulated 4.4-, 6.0-, and 10.4-fold more radioactivity than did EJ/anti-DNS tumors at 4, 24, and $48 \mathrm{~h}$, respectively (Supplemental Fig. 2). Though slight signals could initially be detected in the lung, liver, and kidney, these nonspecific signals faded over time whereas specific signals remained conspicuous in the EJ/anti-PEG tumors. Collectively, these results indicate that the anti-PEG reporter, when paired with PEGylated probes, is useful for noninvasive imaging in vivo by optical imaging, MRI, and PET. 
Comparisons Between Anti-PEG and HSV-tk Reporters

HSV-tk is currently regarded as a gold standard reporter gene. We therefore sought to compare the imaging specificity, immunogenicity, and probe toxicity of the anti-PEG and HSV-tk reporters. EJ/TK was generated by retroviral transduction. Western blotting indicated that EJ/anti-PEG and EJ/TK cells expressed similar levels of anti-PEG or HSV-tk reporter proteins (Supplemental Fig. 3). BALB/c nude mice bearing EJ/anti-PEG and EJ/TK tumors in their right and left hind legs were intravenously injected with 4-arm ${ }^{124}$ I-PEG-SHPP or ${ }^{124}$ I-FIAU. Specific targeting of radioiodinated probes was analyzed by small-animal PET. Figure 3 shows that 4 -arm ${ }^{124}$ I-PEG-SHPP selectively accumulated in EJ/anti-PEG tumors but not in EJ/TK tumors whereas ${ }^{124}$ I-FIAU displayed the opposite specificity. Importantly, imaging specificity attained by the anti-PEG reporter was comparable to HSV-tk.

The immunogenicity of the anti-PEG and HSV-tk reporters was examined after hydrodynamic-based gene transfer of anti-PEG- or HSV-tk-encoding plasmids to $\mathrm{BALB} / \mathrm{c}$ mice. We did not detect noticeable antibody titer in mice injected with anti-PEG-encoding plasmids (Fig. 4). In line with previous reports (3), however, antibodies against HSV-tk were readily detected in the mice injected
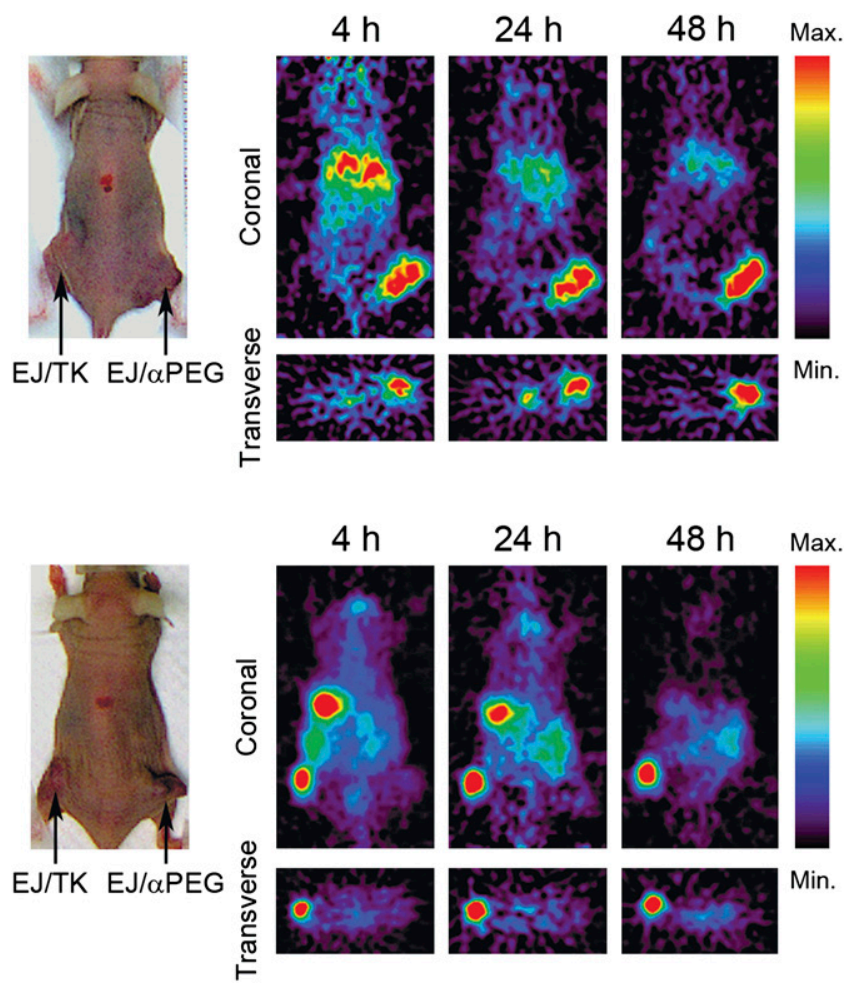

FIGURE 3. Imaging comparison of anti-PEG and HSV-tk reporters. Mice bearing established EJ/anti-PEG (right hind legs) and EJ/TK (left hind legs) tumors were intravenously injected with $3,700 \mathrm{kBq}$ of 4 -arm ${ }^{124}$ I-PEG-SHPP (top) or ${ }^{124}$ I-FIAU (bottom). Small-animal PET images were acquired in coronal planes and transverse planes at 4,24 , and $48 \mathrm{~h}$ after injection of probes. $\alpha P E G=$ anti-PEG. with HSV-tk-encoding plasmids, indicating that HSV-tk was indeed immunogenic.

To assess the safety of anti-PEG or HSV-tk imaging probes, we incubated iodinated 4-arm PEG-SHPP or FIAU with parental EJ, EJ/anti-PEG, EJ/TK, and CNL cells. Consistent with previous results indicating that PEG is a nontoxic polymer (14), iodinated 4-arm PEG-SHPP $(\leq 125 \mu \mathrm{M})$ did not affect the survival of CNL, EJ, or EJ/ anti-PEG cells (Supplemental Fig. 4A). In contrast, FIAU was considerably cytotoxic to CNL cells (inhibitory concentration of $50 \%, 65.9 \mu \mathrm{M}$ ). Moreover, FIAU induced more pronounced cytotoxicity to the cells that expressed HSV-tk (inhibitory concentration of 50\%, 0.18 $\mu \mathrm{M}, P<$ 0.00001, comparing EJ/TK to EJ; Supplemental Fig. 4B). These results and previous reports $(15,16)$ firmly demonstrate that PEGylated probes display little toxicity to mammalian cells. On the contrary, the dose of HSV-tk probes should be carefully adjusted to prevent toxicity.

\section{In Vivo Imaging of Humanized Anti-PEG Reporter}

Before the anti-PEG reporter can be used in humans, the mouse-derived sequences must be humanized to minimize human antimouse immune responses. The complementarity-determining regions of the anti-PEG $\mathrm{V}_{\mathrm{H}}$ and $\mathrm{V}_{\mathrm{L}}$ were grafted onto the human germline DP-35 + JH4 and A2c + JK2 segments to create humanized anti-PEG $V_{H}$ and $V_{L}$ (Supplemental Fig. 5). The humanized anti-PEG $\mathrm{V}_{\mathrm{H}}$ and $\mathrm{V}_{\mathrm{L}}$ genes were fused to human $\operatorname{IgM~} \mathrm{C}_{\mathrm{H}} 1$ and $\mathrm{C}_{\kappa}$ genes to construct the heavy chain and light chain of the humanized anti-PEG reporter (human anti-PEG). The human anti-PEG reporter was functional as shown by binding of PEGquantum dot to EJ/human anti-PEG cells (Fig. 5A). As a test of its in vivo utility as a reporter gene, BALB/c nude mice bearing established EJ/human anti-PEG and EJ/antiDNS tumors in their right and left hind legs, respectively, were intravenously injected with 4-arm ${ }^{124}$ I-PEG-SHPP and then imaged with a small-animal PET scanner. Figure $5 \mathrm{~B}$ shows that specific signals selectively accumulated at EJ/human anti-PEG tumors at $4 \mathrm{~h}$ after injection. The radiointensity of the region of interest was 4.5-, 5.0-, and 10.4-fold greater in the EJ/human anti-PEG tumors than in the EJ/anti-DNS tumors at 4, 24, and $48 \mathrm{~h}$, respectively (similar to EJ/anti-PEG tumors vs. EJ/anti-DNS tumors). In addition, a metastatic lung model of Hela/human anti-PEG cells was also monitored by optical imaging. Figure 5C shows that specific fluorescent signals were detected in the lung foci after intravenous injection of Hela/human antiPEG cells but not control Hela cells. Collectively, these results indicate that humanized anti-PEG may be an optimal reporter gene for noninvasive imaging of gene expression and cell delivery in humans.

\section{DISCUSSION}

Here we report a versatile and universal anti-PEG reporter to trap a wide range of PEGylated imaging probes. Selective retention of PEGylated probes at sites of anti- 


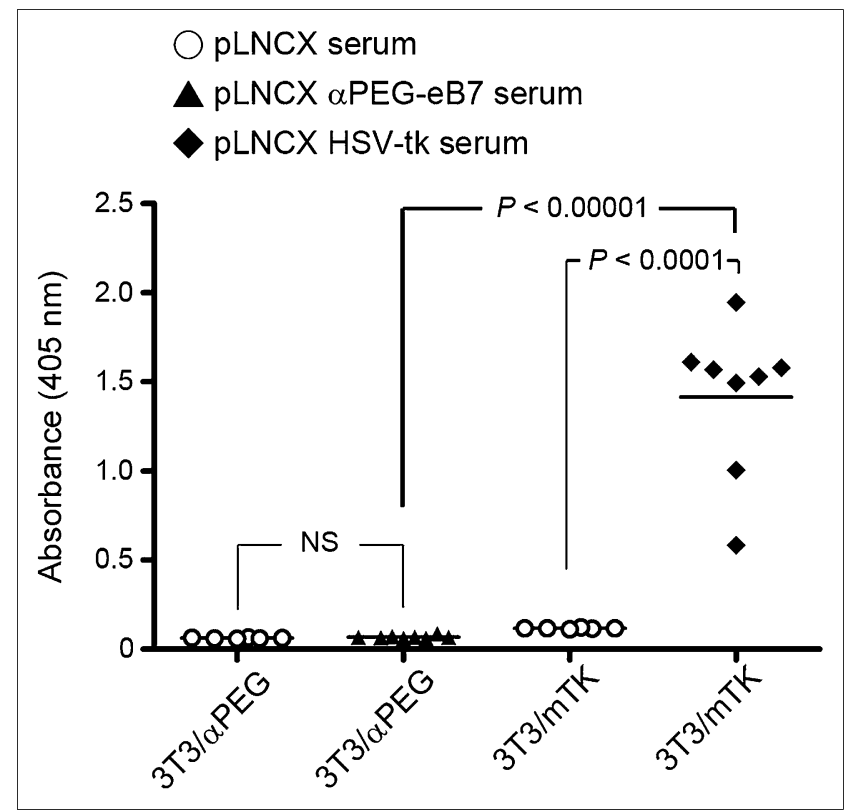

FIGURE 4. Immunogenicity of anti-PEG and HSV-tk reporter systems. Serum samples collected from BALB/c mice $10 \mathrm{~d}$ after hydrodynamic-based gene transduction were assayed by enzyme-linked immunosorbent assay for presence of antibodies against anti-PEG or HSV-tk proteins expressed on surface of 3T3 fibroblasts. Representative data from 3 independent experiments are shown. Horizontal bars indicate mean antibody titer in each group. $\alpha P E G=$ anti-PEG; $\mathrm{mTK}=$ membrane-anchored HSV-tk.

PEG reporter expression can facilitate monitoring of the delivery and expression of genes or cells in vivo by multiple imaging systems, including optical imaging, MRI, and PET. The anti-PEG reporter displayed an imaging specificity comparable to HSV-tk but did not provoke immune responses or cause apparent toxicity. The humanized antiPEG reporter described here also facilitated effective and specific imaging in vitro and in vivo. Collectively, the antiPEG reporter possesses high specificity and low immunogenicity, which may be useful for clinical optimization of gene or cell therapies in humans.

PEG is a nontoxic and biocompatible polymer that has been approved by the Food and Drug Administration for human intravenous, oral, and dermal applications (14). Thus, increasing numbers of PEGylated molecules are in late-stage clinical trials or already approved for human use. In addition, PEG-coated liposomal doxorubicin (25) and PEG Interferon alfa-2b (26) can more effectively control diseases while reducing the injection frequency. As the number of PEGylated molecules expands, the anti-PEG reporter should help their rapid clinical translation.

The development of a versatile reporter that can be monitored by multiple imaging systems will extend its utility in biomedical research. Each imaging modality has inherent strengths and weaknesses. For example, nuclear imaging is highly sensitive but suffers from poor spatial resolution (27). MRI has spectacular image resolution, but imaging sensitivity is inferior to that of nuclear techniques (28). Optical imaging is relatively inexpensive but can be limited by the shallow depth penetration of the signal (29). Because PEG can easily be linked to a wide variety of imaging probes (30), the anti-PEG reporter allows flexibility in choosing the optimal imaging system. In addition, the anti-PEG reporter can efficiently bind even low concentrations of PEGylated probes (1 nM for PEGquantum dots), as long as the PEG chains are greater than $1.5 \mathrm{kDa}(13)$.

Efficient surface expression of the functional anti-PEG reporter can be attributed to 3 main factors. First, a Fab fragment rather than single-chain antibody was used. In most cases, an intact Fab fragment maintains higher binding affinity than a single-chain antibody (31), because interactions across the $\mathrm{V}_{\mathrm{L}} / \mathrm{V}_{\mathrm{H}}$ and $\mathrm{C}_{\mathrm{K}} / \mathrm{C}_{\mathrm{H}} 1$ domains can form a more stable structure than those between $\mathrm{V}_{\mathrm{L}}$ and $\mathrm{V}_{\mathrm{H}}$ domains alone (32). Second, following a previously reported method (20), we inserted furin-2A cleavage sites between the light- and heavy-chain genes to achieve efficient surface expression of the mature anti-PEG reporter from a single open reading frame. The light chain can be liberated from the nascent polypeptide to pair-through disulfide bonds - with the heavy chain to form a functional anti-PEG reporter in the endoplasmic reticulum. Third, the anti-PEG reporter is fused to the transmembrane domain and cytoplasmic tail of the B7-1 antigen, which was found to mediate rapid transport of chimeric protein to the cell surface (33).

To increase the clinical utility of the reporter, we humanized the anti-PEG Fab to minimize human antimouse immune responses and thus allow repeated and persistent imaging of the reporter in humans. Importantly, humanization of the anti-PEG reporter did not hamper its activity; the humanized anti-PEG reporter displayed high imaging specificity in subcutaneous and metastatic tumor models in vivo as determined by smallanimal PET and optical imaging. A wide range of transmembrane domains derived from human proteins including the human B7-1 antigen have been used to anchor heterologous proteins on cells (9), which will facilitate complete humanization of the human anti-PEG reporter. The human anti-PEG reporter, the first example of a humanized antibody used as a reporter gene, may provide a powerful tool for monitoring the efficacy of gene or cell therapy and survival of organ transplants in humans by multiple imaging systems.

The anti-PEG reporter may have widespread use in clinical and experimental medicine. The anti-PEG reporter may be transgenically expressed in specific tissues or organs to allow noninvasive imaging of disease progression. Alternatively, a transgenic mouse that ubiquitously expresses the anti-PEG reporter may serve as a syngeneic cell, tissue, or organ donor to study transplant survival in recipient (nontransgenic) mice by noninvasive imaging. Unlike other transgenic mouse models that use exoge- 
A

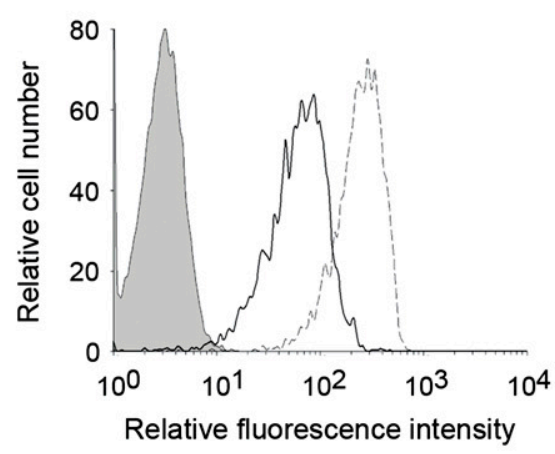

B

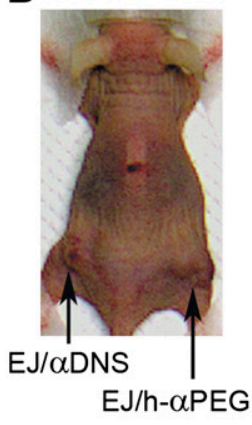

$4 \mathrm{~h}$

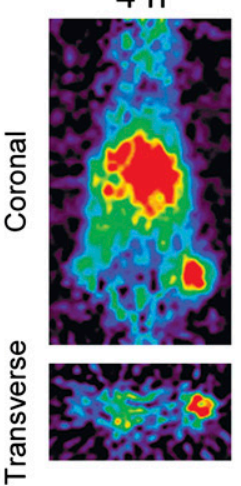

$24 \mathrm{~h}$

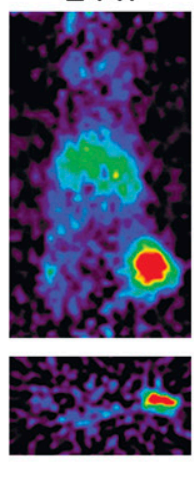

$48 \mathrm{~h}$

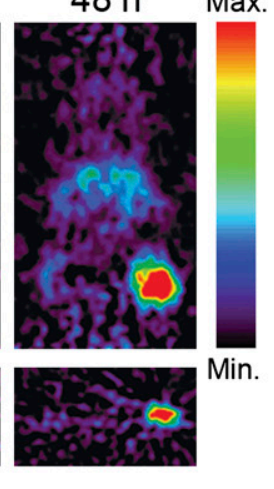

C

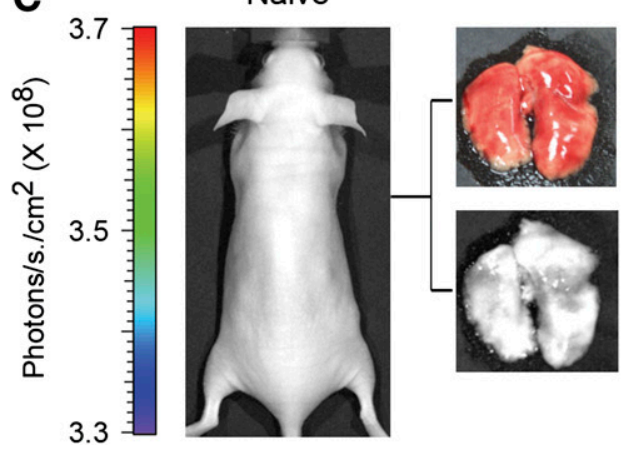

Hela

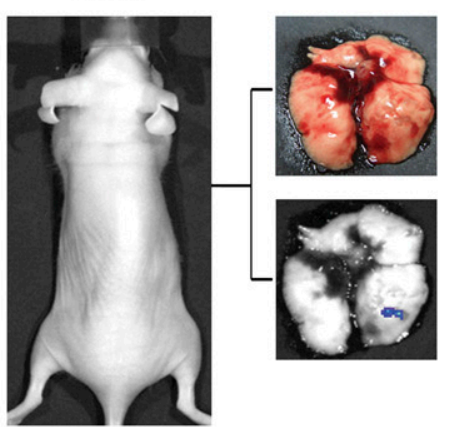

Hela/h- $\alpha$ PEG

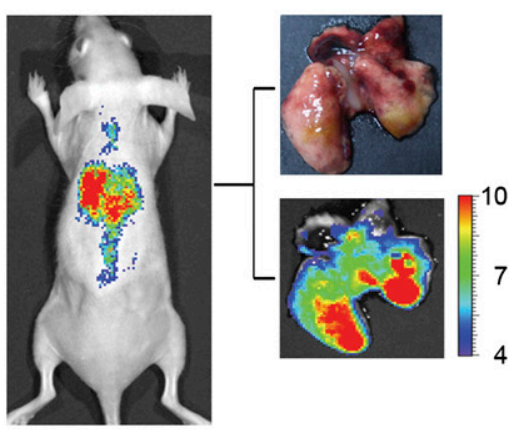

FIGURE 5. Analysis and imaging of humanized anti-PEG reporter. (A) Flow cytometric analysis of surface expression (dashed line, stained by hemagglutinin antibody) and function (solid line, stained by PEG-quantum dot) of human anti-PEG reporter in EJ cell line. Shaded area of graph shows mock staining with PBS. (B) In vivo small-animal PET of mice bearing EJ/human anti-PEG tumors (right hind legs) and EJ/anti-DNS tumors (left hind legs) at 4, 24, and $48 \mathrm{~h}$ after injection of 4-arm ${ }^{124}$ I-PEG-SHPP. Smallanimal PET images were acquired in coronal and transverse planes. Representative images of 3 independent experiments $(n=$ 3) are shown. (C) In vivo optical imaging of naive mice (left panel) or mice bearing Hela (middle panel) and Hela/human anti-PEG (right panel) lung metastatic tumors at $48 \mathrm{~h}$ after injection of PEG-NIR797. Lung tissue was also harvested to confirm tumor foci and specific fluorescent signals. Representative images of 3 independent experiments $(n=3)$ are shown. h- $\alpha P E G=$ human anti-PEG; $\alpha$ DNS = anti-DNS.

nously derived reporters (34), the anti-PEG reporter should not provoke immunity in nontransgenic recipients. Finally, the anti-PEG reporter may be useful for tracking the homing, migration, turnover, and transdifferentiation of stem cells on transplantation.

\section{CONCLUSION}

The anti-PEG reporter system possesses several advantages. The system has high affinity and specificity to allow stable retention of the probes at anti-PEG-expressing sites. The anti-PEG or human anti-PEG reporter can circumvent immune responses to allow repeated and persistent imaging of gene expression in mice or humans. PEG is a safe polymer approved by the Food and Drug Administration and can easily be linked to any suitable imaging probe to pair with multiple imaging systems. Because of these advantages, we believe that the anti-PEG reporter may provide a valuable tool to image the location and delivery of gene therapy or cell therapy, the progression of autoimmune diseases, and the survival of organ transplants in animals and humans.

\section{ACKNOWLEDGMENTS}

This study was supported by grants from the National Health Research Institute, Taipei, Taiwan (NHRI-EX989624SI), and Academia Sinica (AS-98-TP-B09). We acknowledge technical support from the Functional and Micro-Magnetic Resonance Imaging Center and the Molecular and Genetic Imaging Core supported by the National Research Program for Genomic Medicine, National Science Council, Taiwan (NSC98-3112-B-001), the Kaohsiung Medical University Hospital Cancer Center (DOH99-TDC-111-002), and the National Sun Yat-Sen UniversityKaohsiung Medical University Joint Research Center.

\section{REFERENCES}

1. Tai JH, Nguyen B, Wells RG, et al. Imaging of gene expression in live pancreatic islet cell lines using dual-isotope SPECT. J Nucl Med. 2008;49:94-102.

2. Tjuvajev JG, Doubrovin M, Akhurst $\mathrm{T}$, et al. Comparison of radiolabeled nucleoside probes (FIAU, FHBG, and FHPG) for PET imaging of HSV1-tk gene expression. J Nucl Med. 2002;43:1072-1083.

3. Bonini C, Ferrari G, Verzeletti S, et al. HSV-TK gene transfer into donor lymphocytes for control of allogeneic graft-versus-leukemia. Science. 1997;276: 1719-1724. 
4. Johnson AA, Ray AS, Hanes J, et al. Toxicity of antiviral nucleoside analogs and the human mitochondrial DNA polymerase. J Biol Chem. 2001;276:40847-40857.

5. MacLaren DC, Gambhir SS, Satyamurthy N, et al. Repetitive, non-invasive imaging of the dopamine D2 receptor as a reporter gene in living animals. Gene Ther. 1999;6:785-791.

6. Weissleder R, Moore A, Mahmood U, et al. In vivo magnetic resonance imaging of transgene expression. Nat Med. 2000;6:351-355.

7. Wolf G. Vitamin A functions in the regulation of the dopaminergic system in the brain and pituitary gland. Nutr Rev. 1998;56:354-355.

8. Fleming RE, Migas MC, Holden CC, et al. Transferrin receptor 2: continued expression in mouse liver in the face of iron overload and in hereditary hemochromatosis. Proc Natl Acad Sci USA. 2000;97:2214-2219.

9. Cheng TL, Roffler S. Membrane-tethered proteins for basic research, imaging, and therapy. Med Res Rev. 2008;28:885-928.

10. Roffler SR, Wang HE, Yu HM, et al. A membrane antibody receptor for noninvasive imaging of gene expression. Gene Ther. 2006;13:412-420.

11. Wei LH, Olafsen T, Radu C, et al. Engineered antibody fragments with infinite affinity as reporter genes for PET imaging. J Nucl Med. 2008;49:1828-1835.

12. Northrop JP, Bednarski M, Barbieri SO, et al. Cell surface expression of single chain antibodies with applications to imaging of gene expression in vivo. Eur $J$ Nucl Med Mol Imaging. 2003;30:1292-1298.

13. Cheng TL, Cheng CM, Chen BM, et al. Monoclonal antibody-based quantitation of poly(ethylene glycol)-derivatized proteins, liposomes, and nanoparticles. Bioconjug Chem. 2005;16:1225-1231.

14. Fu J, Fiegel J, Krauland E, Hanes J. New polymeric carriers for controlled drug delivery following inhalation or injection. Biomaterials. 2002;23:4425-4433.

15. Leiner T, Ho KY, Ho VB, et al. Multicenter phase-II trial of safety and efficacy of NC100150 for steady-state contrast-enhanced peripheral magnetic resonance angiography. Eur Radiol. 2003;13:1620-1627.

16. Ballou B, Lagerholm BC, Ernst LA, Bruchez MP, Waggoner AS. Noninvasive imaging of quantum dots in mice. Bioconjug Chem. 2004;15:79-86.

17. Mohs AM, Wang X, Goodrich KC, Zong Y, Parker DL, Lu ZR. PEG-gpoly(GdDTPA-co-L-cystine): a biodegradable macromolecular blood pool contrast agent for MR imaging. Bioconjug Chem. 2004;15:1424-1430.

18. Tzou SC, Roffler S, Chuang KH, et al. Micro-PET imaging of betaglucuronidase activity by the hydrophobic conversion of a glucuronide probe. Radiology. 2009;252:754-762.

19. Cheng TL, Wu PY, Wu MF, Chern JW, Roffler SR. Accelerated clearance of polyethylene glycol-modified proteins by anti-polyethylene glycol IgM. Bioconjug Chem. 1999;10:520-528.

20. Fang J, Qian JJ, Yi S, et al. Stable antibody expression at therapeutic levels using the 2A peptide. Nat Biotechnol. 2005;23:584-590.
21. Morrison SL, Porter SB, Trinh KR, Wims LA, Denham J, Oi VT. Variable region domain exchange influences the functional properties of IgG. J Immunol. 1998; 160:2802-2808.

22. Su YC, Chuang KH, Wang YM, et al. Gene expression imaging by enzymatic catalysis of a fluorescent probe via membrane-anchored beta-glucuronidase. Gene Ther. 2007;14:565-574.

23. Yoon SO, Lee TS, Kim SJ, et al. Construction, affinity maturation, and biological characterization of an anti-tumor-associated glycoprotein-72 humanized antibody. J Biol Chem. 2006;281:6985-6992.

24. Kabat EA, Wu TT, Perry HM, Gottesman KS, Foeller C. Sequences of Proteins of Immunological Interest. 5th ed. Bethesda, MD: U.S. Department of Health and Human Services, Public Health Service, National Institutes of Health; 1991:103-511.

25. Ranson MR, Cheeseman S, White S, Margison J. Caelyx (stealth liposomal doxorubicin) in the treatment of advanced breast cancer. Crit Rev Oncol Hematol. 2001;37:115-120.

26. Bukowski RM, Tendler C, Cutler D, Rose E, Laughlin MM, Statkevich P. Treating cancer with PEG Intron: pharmacokinetic profile and dosing guidelines for an improved interferon-alpha-2b formulation. Cancer. 2002;95: 389-396.

27. Madsen MT, Park CH. Enhancement of SPECT images by Fourier filtering the projection image set. J Nucl Med. 1985;26:395-402.

28. Yeung HW, Macapinlac H, Karpeh M, Finn RD, Larson SM. Accuracy of FDGPET in gastric cancer: preliminary experience. Clin Positron Imaging. 1998;1: 213-221.

29. Weissleder R, Ntziachristos V. Shedding light onto live molecular targets. Nat Med. 2003;9:123-128.

30. Roberts MJ, Bentley MD, Harris JM. Chemistry for peptide and protein PEGylation. Adv Drug Deliv Rev. 2002;54:459-476.

31. Schodin BA, Kranz DM. Binding affinity and inhibitory properties of a single-chain anti-T cell receptor antibody. J Biol Chem. 1993;268:2572225727.

32. Rothlisberger D, Honegger A, Pluckthun A. Domain interactions in the Fab fragment: a comparative evaluation of the single-chain $\mathrm{Fv}$ and Fab format engineered with variable domains of different stability. J Mol Biol. 2005;347: 773-789.

33. Liao KW, Chou WC, Lo YC, Roffler SR. Design of transgenes for efficient expression of active chimeric proteins on mammalian cells. Biotechnol Bioeng. 2001;73:313-323.

34. Bubnic SJ, Nagy A, Keating A. Donor hematopoietic cells from transgenic mice that express GFP are immunogenic in immunocompetent recipients. Hematology. 2005;10:289-295. 